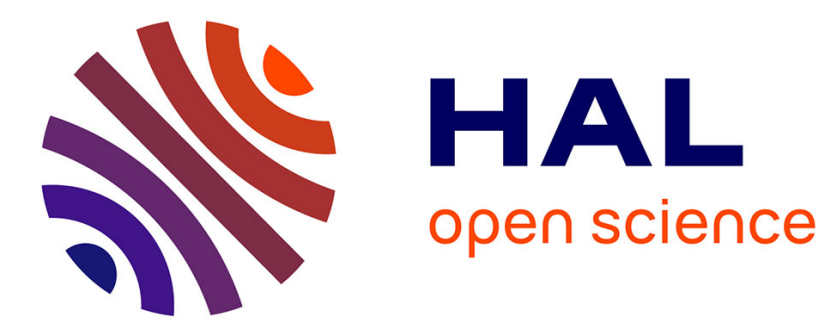

\title{
Réalisation d'une sonde électronique sous hypervide
}

\author{
Alain Nouailhat, Pierre Pinard, Francois Davoine
}

\section{To cite this version:}

Alain Nouailhat, Pierre Pinard, Francois Davoine. Réalisation d'une sonde électronique sous hypervide. Revue de Physique Appliquée, 1967, 2 (3), pp.163-166. 10.1051/rphysap:0196700203016300 . jpa-00242784

\section{HAL Id: jpa-00242784 https://hal.science/jpa-00242784}

Submitted on 1 Jan 1967

HAL is a multi-disciplinary open access archive for the deposit and dissemination of scientific research documents, whether they are published or not. The documents may come from teaching and research institutions in France or abroad, or from public or private research centers.
L'archive ouverte pluridisciplinaire HAL, est destinée au dépôt et à la diffusion de documents scientifiques de niveau recherche, publiés ou non, émanant des établissements d'enseignement et de recherche français ou étrangers, des laboratoires publics ou privés. 


\title{
RÉALISATION D’UNE SONDE ÉLEGTRONIQUE SOUS HYPERVIDE
}

\author{
Par Alain NOUAilhat, Pierre PinARD et François DAVOINe, \\ Institut National des Sciences Appliquées de Lyon, \\ Laboratoire de Physique de la Matière.
}

Résumé. - Dans le but d'étudier, de manière quantitative, certaines interactions électronsmatière, nous avons construit une sonde électronique fonctionnant en ultra-vide.

Les premiers résultats expérimentaux sont relatifs à la mesure, en régime pulsé, des phénomènes rapides de cathodoluminescence du sulfure de cadmium et des halogénures alcalins.

Abstract. - We have built an electron probe in order to make, in ultra high vacuum, a quantitative study of some electron-lattice interactions.

The first experimental results relate to the measurement, in pulsed working, of fast cathodoluminescence phenomena in cadmium sulfide and alkali halides.

1. Introduction. - Le comportement de monocristaux de sulfure de cadmium et d'halogénures alcalins sous irradiation électronique pulsée a été étudié au laboratoire :

- soit de manière purement qualitative au moyen d'un microscope à balayage [1],
- soit quantitativement [2] en analysant les évolutions de la lumière émise au moment de l'établissement et de l'arrêt de chaque excitation.

Ces travaux ont été effectués sous un vide classique (de l'ordre de $10^{-5} \mathrm{~mm}$ de mercure) et avec des énergies primaires inférieures à $20 \mathrm{keV}$.

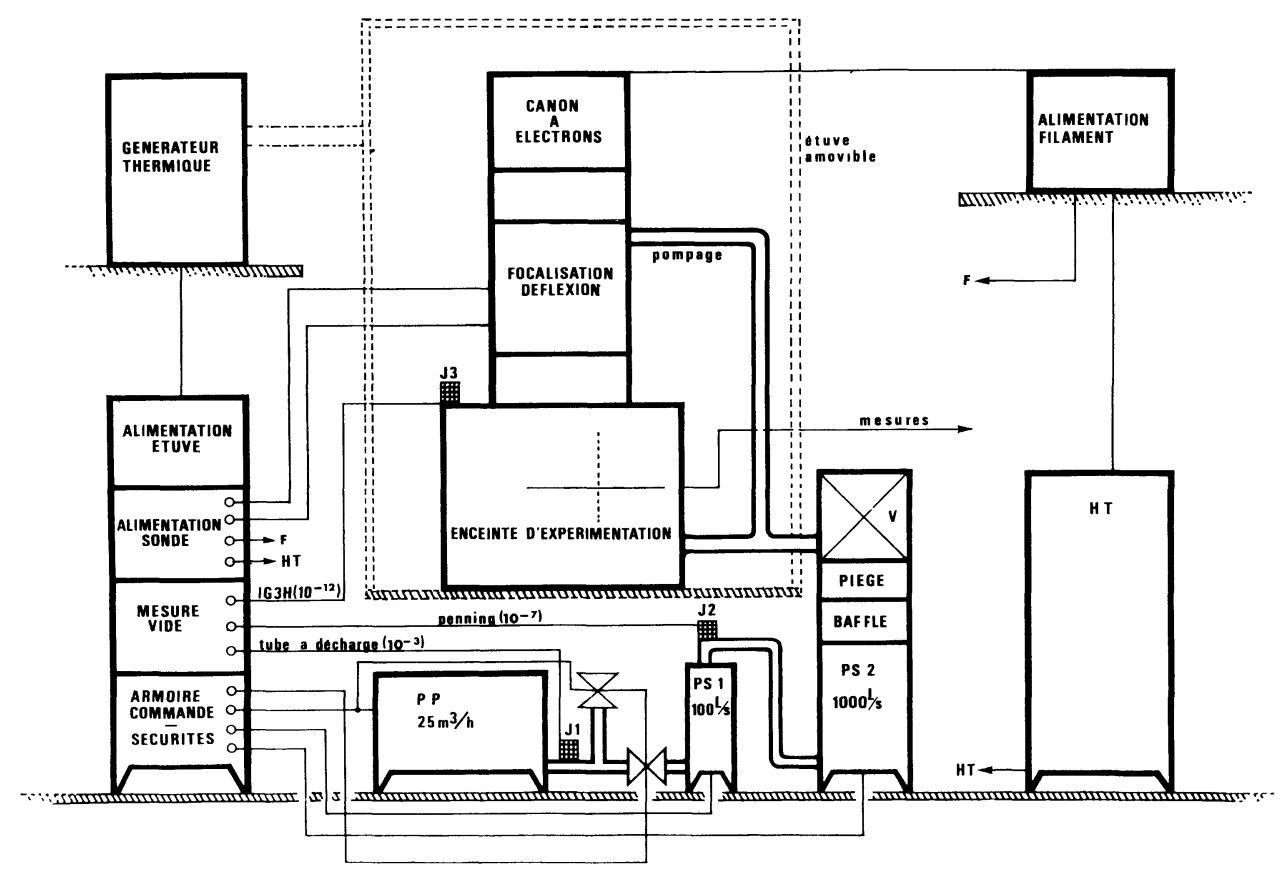

FIG. 1. 
Dans le but de compléter et d'améliorer la précision des résultats déjà obtenus, nous avons réalisé une sonde électronique permettant, sous hypervide $\left(10^{-9} \mathrm{~mm}\right.$ de mercure), de faire varier, dans de larges limites, les conditions d'excitation électronique de l'objet. Cellesci sont les suivantes :

- tension d'accélération : 10 à $150 \mathrm{kV}$,

— intensité : comprise entre 0 et $1 \mu \mathrm{A}$,

- densité électronique incidente : 0 à $10^{-2} \mathrm{~A} / \mathrm{cm}^{2}$,

- température de l'objet : - 193 à $+20^{\circ} \mathrm{G}$,

- durée des impulsions : 0 à $10^{-6} \mathrm{~s}$.

2. Description de l'installation. - Le dispositif expérimental que nous avons construit comprend, essentiellement, les éléments suivants :

2.1. GRoupe DE POMPAGE Ultra-vide. - Le groupe de pompage ultra-vide, représenté par la figure 1 , est constitué d'une pompe primaire de $25 \mathrm{~m}^{3} / \mathrm{h}$ et de deux pompes à diffusion d'huile en série. Il est muni d'un baffle à circulation d'eau et d'un piège à azote liquide dans le but d'éviter la rétrodiffusion d'huile. Précisons que l'enceinte peut être isolée de cet ensemble par une vanne à ultra-vide.

Afin d'assurer un pompage permanent, nous avons assisté le tableau de commande du groupe de pompage

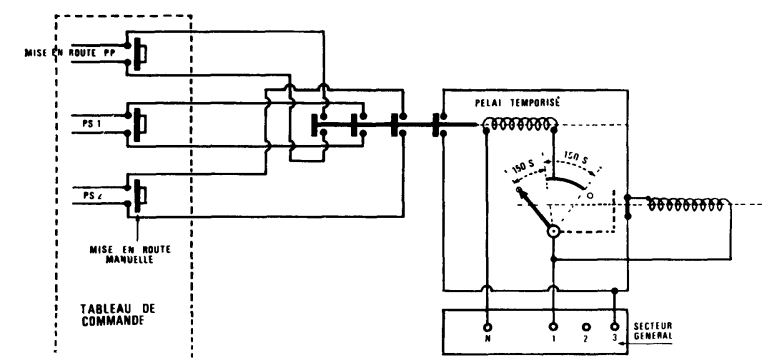

FIG. 2.

de dispositifs électromécaniques ( fig. 2) destinés à mettre ce dernier automatiquement en marche :

- soit en cas de panne de secteur, entraînant l'arrêt général et l'isolement des pompes secondaires de la pompe primaire par la vanne électromagnétique,

- soit en cas de baisse de pression d'eau provoquant le déclenchement des bilames thermiques des pompes à diffusion.
Toutefois, la remise en route ne se produit que si la durée de l'un ou de l'autre de ces arrêts n'excède pas 300 secondes.

2.2. Dispositif D'étuvage. - Pour être en mesure d'étuver l'ensemble de l'appareil, toutes les pièces formant l'enveloppe sont, soit en acier inoxydable poli intérieurement, soit en verre ou en alumine préalablement dégazée sous vide à $2000^{\circ} \mathrm{C}$. Par ailleurs, l'étanchéité est obtenue au moyen de joints d'or, les communications électriques s'effectuant par l'intermédiaire de passages " verre-métal ».

L'étuvage de la vanne et du piège est réalisé grâce à des colliers chauffants. Quant à celui de l'enceinte à ultra-vide elle-même, il est assuré par une circulation en circuit fermé d'air chaud. Celui-ci, issu d'un générateur thermique d'une puissance maximum de $9 \mathrm{~kW}$, est envoyé à l'intérieur d'une étuve amovible formée de deux demi-coquilles qui viennent coiffer l'enceinte.

Un régulateur thermique maintient la température à la valeur désirée et son repérage s'effectue au moyen de thermocouples placés à l'intérieur de l'étuve.

Il faut noter que la présence de nombreux appareillages internes accroît considérablement la durée de l'étuvage : par exemple, on ne peut atteindre une pression inférieure à $10^{-9} \mathrm{~mm}$ de mercure qu'à la suite d'un chauffage, à $300^{\circ} \mathrm{G}$, d'une durée de $24 \mathrm{~h}$.

2.3. Sonde élegtronique. - a) Canon à électrons. - Le canon du type Bruck et Bricka [3] que nous avons construit convient pour des potentiels accélérateurs compris entre 10 et $150 \mathrm{kV}$. Il comprend un filament de tungstène en épingle à cheveux supporté par une plaquette de stéatite. La position de sa pointe et son enfoncement dans le wehnelt sont réglables de l'extérieur, au moyen de 3 poussoirs micrométriques à $120^{\circ}$. Le wehnelt est un cylindre de cuivre. Le trou assurant le passage et la focalisation des électrons a un diamètre de $1,2 \mathrm{~mm}$.

Comme le montre la figure 3, le filament est alimenté par courant alternatif, redressé et filtré, réglable au niveau basse tension par rototransformateur, la liaison BT-HT étant assurée par un transformateur isolé à $150 \mathrm{kV}$.

Précisons qu'en fonctionnement nous nous plaçons toujours en régime de charge d'espace, de sorte que l'intensité du faisceau électronique est parfaitement stable.

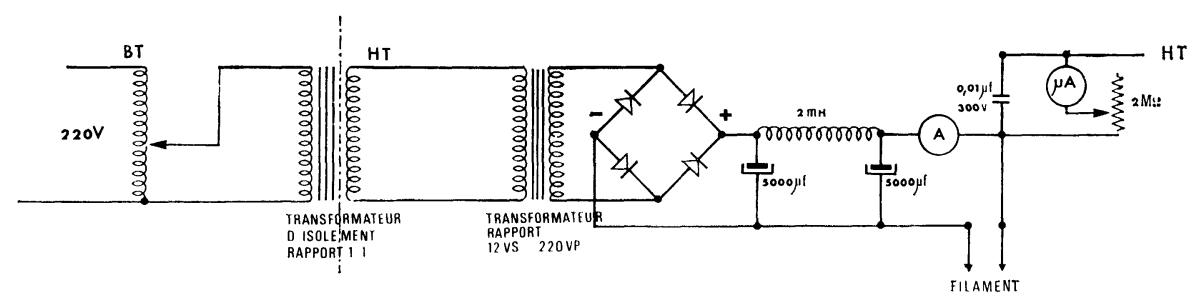

FIG. 3. 
b) Ensemble de focalisation et de déflexion. - Cet ensemble rigide peut être centré au moyen de trois poussoirs micrométriques disposés à $120^{\circ}$.

La lentille magnétique, calculée suivant des critères fournis par Durandeau [4], possède une distance focale de $100 \mathrm{~mm}$ pour une tension primaire égale à $150 \mathrm{kV}$. Les bobinages, de résistance totale $600 \Omega$, sont en fil émaillé de $0,2 \mathrm{~mm}$ de diamètre, afin de pouvoir supporter une température supérieure à $300^{\circ} \mathrm{C}$. La lentille est gainée par une chemise étanche en acier inoxydable amagnétique de manière à éviter, en hypervide, le dégazage de ses enroulements. En régime permanent, sa température interne à pleine puissance ( 6 watts) s'élève à environ $50^{\circ} \mathrm{C}$ au-dessus de la température ambiante.

Les plaques de déflexion électrostatique, attaquées par des signaux sinusoïdaux, en dents de scie, ou carrés, sont solidaires de la lentille et centrées par construction par rapport à l'axe de celle-ci.

Cette sonde électronique fournit un faisceau analyseur dont l'intensité est au plus égale à $1 \mu \mathrm{A}$. Le spot ayant un diamètre de $100 \mu$, il en résulte que l'échantillon pourra être soumis à une densité électronique maximum de $10^{-2} \mathrm{~A} / \mathrm{cm}^{2}$.

2.4. Enceinte D'eXPÉRIMENTATION. - Son volume utile est de $40 \mathrm{dm}^{3}$. Elle comprend actuellement :

- d'une part, un porte-objet tournant avec les dispositifs de mesure et de contrôle des caractéristiques du spot analyseur,

- d'autre part, une paire de plaques de déflexion électrostatique situées au-dessus du porte-objet qui compensent, pour les faibles tensions d'accélération $(V<20 \mathrm{kV})$, la déviation provenant du champ magnétique terrestre.

Notons enfin que le porte-objet est visible de l'extérieur grâce à un système de deux miroirs plans.

Description du porte-objet. — La figure 4 schématise le porte-objet tournant " à barillet » que nous avons construit.

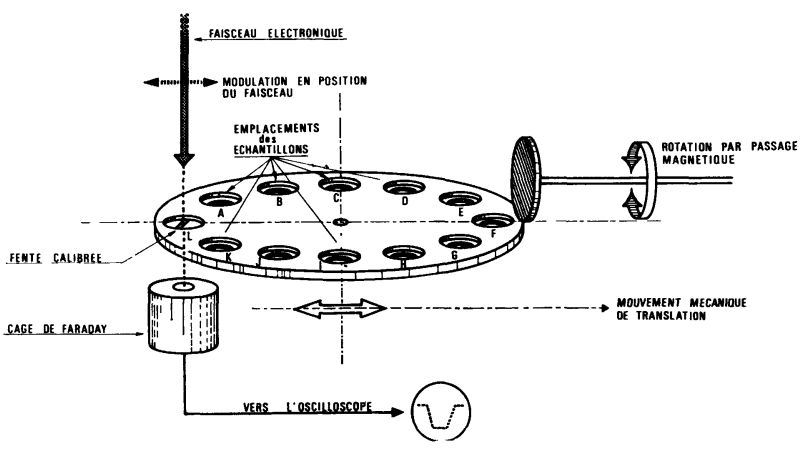

FIG. 4.

On réalise, au moyen d'un passage magnétique, la rotation du disque support d'échantillons afin de pouvoir placer ceux-ci, les uns après les autres, sous le faisceau incident. De plus, la possibilité de faire subir au disque support une translation rectiligne orthogonale au mouvement circulaire permet d'obtenir un positionnement très précis de l'objet.

Pour mesurer, au niveau de l'échantillon, l'intensité et le diamètre du spot électronique, on déplace ce dernier, en agissant sur les plaques de déflexion, perpendiculairement à une fente calibrée tenant lieu d'objet et sous laquelle se trouve une cage de Faraday. L'observation à l'oscilloscope du courant ainsi recueilli fournit les grandeurs recherchées.

3. Quelques résultats expérimentaux. - Après avoir effectué les mises au point indispensables ainsi que diverses expériences préliminaires, nous avons entrepris la détermination précise des régimes transitoires en cathodoluminescence. Mais, par suite du grand nombre de facteurs susceptibles d'intervenir, nous nous sommes limités, volontairement pour le moment, à l'étude du comportement de monocristaux de sulfure de cadmium et d'halogénures alcalins à la température ambiante.

En outre, dans le but de recouper des expériences déjà réalisées au laboratoire, les résultats expérimentaux décrits ci-dessous ont été obtenus au moyen d'un faisceau d'électrons dont les caractéristiques sont les suivantes :

- tension accélération : $12 \mathrm{kV}$,

— intensité : $0,5 \mu \mathrm{A}$,

- densité : $10^{-2} \mathrm{~A} / \mathrm{cm}^{2}$,

le régime pulsé étant créé avec un signal carré de fréquence $5000 \mathrm{~Hz}$ (temps de montée voisin de $10^{-7} \mathrm{~s}$ ).

Le dispositif analyseur est constitué d'un photomultiplicateur muni, éventuellement, de filtres colorés, et d'un oscilloscope. Les mesures s'effectuent sous une pression de l'ordre de $5 \times 10^{-9} \mathrm{~mm}$ de mercure à la suite d'un double cycle d'étuvage à $250^{\circ} \mathrm{C}$.

1. Étude du sulfure de Gadmium. - Dans de telles conditions expérimentales, le monocristal de CdS émet presque exclusivement la bande verte $(5010 \AA)$, cette émission étant localisée au point d'impact.

Si la densité électronique incidente diminue (ce qui, par exemple, s'obtient simplement en défocalisant légèrement le faisceau incident), l'échantillon s'illumine entièrement en rouge.

On met ainsi en évidence la très grande différence d'absorption des longueurs d'ondes rouge et verte par le monocristal de sulfure de cadmium.

Par ailleurs, les temps de montée à mi-hauteur de l'intensité lumineuse émise ont été déterminés et nous avons obtenu les résultats suivants :

$$
\begin{aligned}
& \text { Raie rouge }: t_{1 / 2}=30 \mu \mathrm{s} \\
& \text { Raie verte }: t_{1 / 2}=20 \mu \mathrm{s} .
\end{aligned}
$$


2. Étude des halogénures algalins. - Les temps d'établissement et de déclin de la luminescence sont sensiblement identiques et voisins de $100 \mu$ s avec des substances telles que $\mathrm{KBr}, \mathrm{KGl}$ ou $\mathrm{LiF}$.

$\mathrm{NaCl}$, par contre, possède une plus grande rémanence : $150 \mu$ s.

Enfin, la réponse de l'iodure de potassium est beaucoup plus complexe :
Temps de montée : $t=30 \mu \mathrm{s}$, avec exaltation de la luminescence pendant $100 \mu \mathrm{s}$.

Temps de déclin : $t=100 \mu \mathrm{s}$.

Ces quelques résultats expérimentaux, en parfait accord'avec ceux déjà obtenus [2], montrent l'intérêt de notre dispositif expérimental pour l'étude quantitative des régimes transitoires en cathodoluminescence.

Manuscrit reçu le 9 février 1967.

\title{
BIBLIOGRAPHIE
}

[1] Davoine (F.), Pinard (P.) et Nouailhat (A.), Étude au microscope électronique à balayage de la cathodoluminescence de l'iodure de potassium, J. Microscopie, 1962, 1, 93.

[2] Bechaz (G.), Gauthier (R.), Pinard (P.) et DavOINE (F.), Étude des régimes transitoires de cathodoluminescence, $J$. Microscopie, 1966, 5, 551-568.
[3] BRUCK et BrICKA, Ann. Radioélectricité, 1948, 3, 339 .

[4] Durandeau (P.), Étude sur les lentilles électroniques magnétiques, Thèse de Doctorat ès Sciences, Toulouse, 1957.

\section{SUR LA DÉTERMINATION DES VARIATIONS DE LA PERMÉABILITÉ DIÉLEGTRIQUE GOMPLEXE}

\author{
Par S. IVANOV, D. MICHEV et L. BONTGHEV, \\ Institut de Physique de l'Académie bulgare des Sciences.
}

\begin{abstract}
Résumé. - Dans le présent exposé, on traite de l'appareillage de mesure destiné à effectuer la séparation, l'examen et l'enregistrement des variations des deux composantes de la perméabilité diélectrique complexe de certains semiconducteurs, qui se produisent simultanément à l'éclairement continu et par impulsions.
\end{abstract}

Abstract. - This paper deals with the measuring instrument aimed at carrying out the separation, the study and the recording of the variations of the two components of the complex dielectric permittivity of some semiconductors, simultaneously produced by continuous and pulsed illumination.

A l'éclairement de certains semiconducteurs, insérés comme diélectriques dans un condensateur, on constate que des variations de la perméabilité diélectrique et des pertes diélectriques se produisent simultanément [1]. La séparation des deux composantes de la perméabilité diélectrique complexe a fait l'objet d'une série de travaux scientifiques. Pour résoudre ce problème, on peut suivre plusieurs méthodes : variation de la fréquence d'un générateur dans le circuit oscillant duquel on a inséré le condensateur comportant le semiconducteur en question [2] ; méthode de battement entre un oscillateur à haute stabilité et l'oscillateur contenant l'échantillon inséré dans le circuit [3]; méthodes de pont [4]; Q-mètres [5]; méthodes de résonance [6]; et d'autres. Toutes les méthodes énumérées, cependant, ne donnent pas de solution au problème de la détermination des deux composantes avec la précision suffisante.

Dans certains travaux plus récents, on traite des possibilités du pont phaso-sélectif RLGM [7]. En [8] on a donné le schéma d'un tel dispositif ainsi que les résultats des mesures effectuées sur certains semiconducteurs.

Ces dispositifs ont, eux aussi, des inconvénients; certains influencent plus au moins la précision des résultats des mesures. Ge sont : influence des bruits de fond (surtout provenant du circuit de chauffage) et du courant initial de la diode sur le fonctionnement du détecteur; influence des variations des tensions d'alimentation; manque de possibilité pour des mesures à différentes fréquences; appréciation subjective de la cinétique des processus, et d'autres. 